\title{
Changes in the practice of cardiology: therapies at the forefront of science
}

\author{
Joshua Ping-Yun $\underline{\operatorname{Loh}}^{1,2}$, MRCP, FACC, Kian-Keong $\underline{\text { Poh }}{ }^{1,2}$, FRCP, FACC
}

T he practice of cardiology has evolved significantly over the years. The availability of current diagnostics and therapeutics has enabled physicians to treat increasingly complex cardiovascular diseases (CVDs). Despite this, the burden of CVD is increasing in Southeast Asia, with ischaemic heart disease and cerebrovascular disease accounting for the majority of cardiac-related deaths in this region. ${ }^{(1)}$ To address these unmet needs, we must first acknowledge that Asian-specific data is required to better understand treatment effect on Asian populations, in particular the heterogeneous composition in Southeast Asia. This issue of the Singapore Medical Journal (SM) features a collection of local and Asian original research and review articles, which centres on current and novel approaches to treating CVD.

Managing cardiovascular risk factors is the cornerstone of cardiology practice. In the practice of preventative cardiology, there is an ongoing controversy with regard to managing cholesterol levels to lower cardiovascular risk. In particular, the 2013 American College of Cardiology/American Heart Association Cholesterol Treatment Guidelines adopted a statin-centric approach, disregarding low-density lipoprotein (LDL) goals. ${ }^{(2)}$ Similarly, the revised 2015 American Diabetes Association guidelines also recommended high-intensity statins for diabetic patients. ${ }^{(3)}$ In contrast, the Europeans adopted an LDL-centric approach based on cardiovascular risks. ${ }^{(4)}$ How do we adopt these seemingly differing recommendations in our daily practice? Are the risk prediction models developed based on Western populations accurate in Asian patients? Are Asians more intolerant of high-dose statins? The review article by Yan et al summarises the key findings and recommendations of these various guidelines. ${ }^{(5)}$ The Singapore Ministry of Health $(\mathrm{MOH})$ guidelines on lipids have not yet been published. These two articles (Yan et al and the yet to be published $\mathrm{MOH}$ guidelines) will discuss key principles for effective management of cardiovascular risk locally.

Teong et al focused on the treatment effect of valsartan, an angiotensin receptor blocker (ARB), in a group of hypertensive Asians, as measured using central aortic systolic pressure (CASP). ${ }^{(6)}$ This study used the BPro ${ }^{\circledR}$ (radial pulse wave acquisition device) watch to measure the CASP by capturing radial arterial waveforms at the wrist. ${ }^{(7)}$ The BPro watch, developed by Dr Ting Choon Meng, a Singaporean doctor and an inventor of medical devices, was validated to accurately measure CASP. ${ }^{(8)}$ This study is important to the cardiology community, as CASP has been shown to predict cardiovascular events better than brachial blood pressure. ${ }^{(9)}$ Moreover, ARBs are thought to act more favourably on CASP than other classes of antihypertensive drugs, albeit only in Western populations. ${ }^{(10)}$ This open-label prospective cohort study, which demonstrates the changes in CASP with treatment over 12 weeks in an Asian population, reflects the potential in marrying the use of locally developed medical technology and research to address a knowledge gap in this region.

Another healthcare innovation with high potential is the use of remote monitoring or telehealth. Remote monitoring of blood pressure has been implemented in several healthcare systems in the region, including our institution. This allows time-efficient intervention by healthcare providers without requiring the patient to visit the clinic. An open channel of communication allows the doctor or nurse to titrate hypertensive medications over the phone. Likewise, remote weight monitoring in heart failure patients allows for intervention (e.g. increase in diuretic dose) before the patient goes into acute heart failure and requires hospitalisation. Remote transmission of data from cardiac implantable electronic devices (CIEDs) is now available. These CIEDs include pacemakers, implantable cardioverter defibrillators and cardiac resynchronisation therapy-defibrillators. In a pilot study by Lim et al, 57 patients with CIED were followed up for six months using scheduled wireless remote transmissions, in addition to routine clinic visits. ${ }^{(11)}$ This study highlights the feasibility of such technology to improve patient quality of life, patient safety by earlier detection of problems and compliance to device monitoring. However, the study also underscores the need for proper information filtering in the era of 'big data'. In the study, $98.2 \%$ of transmissions did not contain alerts that required intervention, and of the unscheduled alerts, only $33.3 \%$ required some form of intervention. However, with further streamlining of the healthcare data management process and integration into clinical care, remote monitoring may be a good tool to improve healthcare resource utilisation.

The field of coronary intervention for coronary artery disease has matured considerably in the last two decades. Coronary stents have now replaced balloon angioplasty as the primary mode of treating coronary stenosis. Current-generation drugeluting stents (DES) have been shown to be both efficacious and safe compared to first-generation DES and bare-metal stents in a variety of indications, from stable coronary artery disease to acute coronary syndrome. ${ }^{(12,13)}$ As such, stents have been implanted even in complex lesion subtypes such as left main

${ }^{1}$ Department of Cardiology, National University Heart Centre, National University Health System, ${ }^{2}$ Department of Medicine, Yong Loo Lin School of Medicine, National University of Singapore, Singapore

Correspondence: A/Prof Kian-Keong Poh, Department of Cardiology, National University Heart Centre, National University Health System, 1E Kent Ridge Rd, NUHS Tower Block, Level 9, Singapore 119228. kian_keong_poh@nuhs.edu.sg 
stenosis, bifurcating stenosis, multi-vessel disease and chronic total occlusion. However, contemporary practice calls for a heart-team approach, where a team of interventional cardiologists and cardiac surgeons come together to decide on the optimal approach to revascularisation (whether percutaneous coronary intervention $[\mathrm{PCl}]$, coronary artery bypass grafting or medical therapy) in stable patients with left main or complex multi-vessel disease. ${ }^{(14)}$ In the setting of acute myocardial infarction, $\mathrm{PCl}$, when performed in a timely fashion, results in improved survival. This is especially important in patients presenting with acute ST-segment elevation myocardial infarction, where every ten-minute delay in door-to-reperfusion translates to an $8 \%$ increase in in-hospital mortality. ${ }^{(15)}$

Acute myocardial infarction resulting from left main stenosis or occlusion, although rare, is a clinically catastrophic scenario. Usually a large area of myocardium is infarcted, and most of the patients present with cardiogenic shock. Notwithstanding successful reperfusion (often with $\mathrm{PCl}$ ), the in-hospital mortality is exceedingly high ( $>40 \%$ in many of the known series published). We have limited data on the long-term prognosis of patients who survive the initial episode. In this issue, Xu et al's report of their centre's experience will help in understanding the longer-term outcomes of survivors. ${ }^{(16)}$ In this study, in a mean follow-up period of 44.6 months, the overall mortality was $>50 \%$, with a cumulative survival rate of only $30.7 \%$. Consistent with other case series, in-hospital cardiogenic shock was a predictor of mortality. The authors also rightly pointed out that a large, multicentre, collaborative registry is much needed to further understand the best treatment options in such catastrophic scenarios of low incidence. What is interesting in this report, which perhaps should be further discussed, was that five of the 55 patients (9\%) presented with stent thrombosis of the left main artery (rather than a de novo occlusion). What type of stents were used? Was stent thrombosis due to a failure of antiplatelet therapy, or was it related to inadequate optimisation of the initial left main stenting procedure?

A new frontier of therapeutics in the last decade was the advent of the transcatheter heart valves. Transcatheter aortic valve implantation (TAVI) refers to a percutaneous approach (usually by the femoral artery or an apical approach through a small incision in the ribcage), where a balloon-expandable or selfexpanding valve is implanted within the native aortic valve. TAVI has now become the treatment of choice for patients with severe symptomatic aortic stenosis who are deemed inoperable, and an alternative treatment option to surgical aortic valve replacement in patients at high surgical risk. ${ }^{(17-20)}$ Moreover, a recent analysis indicates that TAVI was noninferior compared with surgery in patients at intermediate surgical risk, ${ }^{(21)}$ with trials under way to evaluate TAVI in patients at low surgical risk.

Further innovations in treatment strategy have led to TAVI being used not only for native aortic valves, but also degenerative bioprosthetic aortic valves. As bioprostheses are increasingly used over mechanical valves in surgical aortic valve replacements, it is anticipated that more patients will present with degenerated bioprostheses. Hence, the valve-in-valve option is proposed for patients who are at an elevated risk of undergoing a reoperation, often due to a combination of advanced age and a scarred chest. A global valve-in-valve registry indicated a one-year survival rate of $83.2 \%$, with $>90 \%$ of patients who survived the first month having good functional status. ${ }^{(22)}$ In this issue, Chiam et al reports the first Asian experience with valve-in-valve TAVI in eight patients, demonstrating no adverse events in the first month, and a one-year survival rate of $87.5 \% .{ }^{(23)}$ This article reports on the feasibility of valve-in-valve TAVI in a selected group of Asian patients.

Another innovation reported in this issue is the use of nonfluoroscopic systems (NFS) to guide radiofrequency catheter ablation (RFCA) for supraventricular tachycardia. See et al evaluated 200 patients who underwent RFCA and demonstrated that patients who underwent RFCA with NFS had lower ionising radiation dose and fluoroscopy time compared to conventional fluoroscopy with similarly high-procedural success. ${ }^{(24)}$ The main limitation, however, was the non-randomised nature of the study. Also, the decision to use NFS over conventional fluoroscopy was left to the operator. What was not entirely clear in the paper was the number of operators involved in the study and the varying skill sets among the operators. Nevertheless, it demonstrates that good patient selection and the usage of suitable techniques should result in better radiation safety for the patients and medical team. This is especially important, as a recent report suggests significantly more frequent health-related problems (including cancer and cataract) among cardiac catheterisation lab workers compared to unexposed controls. ${ }^{(25)}$

Another topic of discussion in this issue relates to heart failure. Due to the global increase in incidence of ischaemic heart disease and survival after myocardial infarction, many patients are now living with heart failure. Heart failure, which has become a pandemic locally and worldwide, leads to significant morbidity and mortality. ${ }^{(26)}$ In the United States, heart failure is the leading cause of hospital admissions. Annually, more than one million patients are admitted for heart failure, with $>50 \%$ readmitted within six months. Heart failure admissions cost the American government a staggering USD 17 billion annually. ${ }^{(27)}$ Managing heart failure requires a multi-pronged approach, including, but not limited to, guideline-directed medical therapy, revascularisation and device therapy.

For acute heart failure, the goal is symptom relief with diuretics, which reverses fluid retention and clinical congestion. In a subset of patients with diuretic resistance, treatment options are extremely limited. Extracorporeal removal of fluids using ultrafiltration has been evaluated as a safe and effective alternative to diuretics in treating acute heart failure. ${ }^{(28)}$ It has, however, not been extensively evaluated in this subset of patients with diuretic resistance. A report in this issue by Teo et al evaluated 44 patients hospitalised with acute heart failure who fulfilled the criteria of diuretic resistance. ${ }^{(29)}$ Compared to the control group who received diuretics, the ultrafiltration group achieved greater fluid and weight loss, a shorter hospital stay, better health status at 30 days, and a reduction in rehospitalisations for heart failure at 90 days. This study has significant implications; on a larger scale, it 
may potentially lead to significant healthcare cost-containment in this difficult-to-manage subset of heart failure patients. The next steps may be for the investigators to expand their study to include multi-Asian centres and perform a randomised controlled trial with cost-effectiveness analysis outcomes.

Lastly, chronic heart failure is usually managed with guideline-directed medical therapy. The role of device therapies as an adjunct therapy is reviewed in an article by Chia and Foo. In particular, the implantable cardioverter defibrillator acts to prevent sudden cardiac death due to ventricular tachyarrhythmia, while cardiac resynchronisation therapy in selected patients acts to reverse pump failure by effectively resynchronising ventricular contractions. ${ }^{(30)}$ Sudden cardiac death and progressive pump failure are the two main causes of death in patients with severe left ventricular dysfunction.

In summary, we have highlighted a spectrum of CVD with treatments at the forefront of science. In this issue of the $S M$ J, it is heartening to see Asian data reported from studies originating from Asia and Singapore, with the potential for future collaborations and further expansion of study concepts. This demonstrates that clinicians and researchers actively seek to meet unmet needs, and bridge the knowledge gap between the East and West in tackling the burden of CVD.

\section{REFERENCES}

1. Tai ES, Poulton R, Thumboo J, et al. An update on cardiovascular disease epidemiology in South East Asia. Rationale and design of the LIFE course study in CARdiovascular disease Epidemiology (LIFECARE). CVD Prev Control 2009; 4:93-102.

2. Stone NJ, Robinson JG, Lichtenstein AH, et al. 2013 ACC/AHA guideline on the treatment of blood cholesterol to reduce atherosclerotic cardiovascular risk in adults: a report of the American College of Cardiology/American Heart Association Task Force on Practice Guidelines. Circulation 2014; 129(25 Suppl 2):S1-45.

3. American Diabetes Association. Standards of Medical Care in Diabetes-2015. Diabetes Care 2015; 38(Suppl 1):S41-S48.

4. Piepoli MF, Hoes AW, Agewall S, et al. 2016 European Guidelines on cardiovascular disease prevention in clinical practice: The Sixth Joint Task Force of the European Society of Cardiology and Other Societies on Cardiovascular Disease Prevention in Clinical Practice (constituted by representatives of 10 societies and by invited experts): Developed with the special contribution of the European Association for Cardiovascular Prevention \& Rehabilitation (EACPR). Eur Heart J 2016 May 23. pii: ehw106 [Epub ahead of print].

5. Yan P, Tan EK, Choo JC, et al. Statin-centric versus low-density lipoproteincentric approach for atherosclerotic cardiovascular disease prevention: a Singapore perspective. Singapore Med J 2016; 57:360-7.

6. Teong $\mathrm{HH}$, Chin AM, Sule AA, Tay JC. Effect of angiotensin receptor blockade on central aortic systolic blood pressure in hypertensive Asians measured using radial tonometry: an open prospective cohort study. Singapore Med J 2016; 57:384-9.

7. BPRO ${ }^{\circledR}$ - Radial Pulse Wave Acquisition Device [online]. Available at: http://www.bpro.ie/. Accessed May 26, 2016.

8. Williams B, Lacy PS, Yan P, et al. Development and validation of a novel method to derive central aortic systolic pressure from the radial pressure waveform using an n-point moving average method. J Am Coll Cardiol $2011 ; 57: 951-61$

9. Williams B, Lacy PS, Thom SM, et al; CAFE Investigators; AngloScandinavian Cardiac Outcomes Trial Investigators; CAFE Steering Committee and Writing Committee. Differential impact of blood pressurelowering drugs on central aortic pressure and clinical outcomes: principal results of the Conduit Artery Function Evaluation (CAFE) study. Circulation 2006; 113:1213-25.

10. Schneider MP, Delles C, Klingbeil AU, et al. Effect of angiotensin receptor blockade on central haemodynamics in essential hypertension: results of a randomised trial. J Renin Angiotensin Aldosterone Syst 2008; 9:49-56.

11. Lim PC, Lee AS, Chua KC, et al. Remote monitoring of patients with cardiac implantable electronic devices: a Southeast Asian, single-centre pilot study. Singapore Med J 2016; 57:372-7.

12. Palmerini $T$, Benedetto $U$, Biondi-Zoccai $G$, et al. Long-term safety of drugeluting and bare-metal stents: evidence from a comprehensive network meta-analysis. J Am Coll Cardiol 2015; 65:2496-507.

13. Palmerini T, Biondi-Zoccai G, Della Riva D, et al. Clinical outcomes with drug-eluting and bare-metal stents in patients with ST-segment elevation myocardial infarction: evidence from a comprehensive network metaanalysis. J Am Coll Cardiol 2013; 62:496-504.

14. American College of Emergency Physicians; Society for Cardiovascular Angiography and Interventions, O'Gara PT, Kushner FG, Ascheim DD, et al. 2013 ACCF/AHA guideline for the management of ST-elevation myocardial infarction: a report of the American College of Cardiology Foundation/American Heart Association Task Force on Practice Guidelines. J Am Coll Cardiol 2013; 61:e78-140.

15. Nallamothu BK, Normand SL, Wang Y, et al. Relation between doorto-balloon times and mortality after primary percutaneous coronary intervention over time: a retrospective study. Lancet 2015; 385:1114-22.

16. Xu L, Sun H, Wang LF, et al. Long-term prognosis of patients with acute myocardial infarction due to unprotected left main coronary artery disease: a single-centre experience over 14 years. Singapore Med J 2016; 57:396-400

17. Makkar RR, Fontana GP, Jilaihawi $\mathrm{H}$, et al; PARTNER Trial Investigators. Transcatheter aortic-valve replacement for inoperable severe aortic stenosis. N Engl J Med 2012; 366:1696-704.

18. Popma JJ, Adams DH, Reardon MJ, et al; CoreValve United States Clinical Investigators. Transcatheter aortic valve replacement using a self-expanding bioprosthesis in patients with severe aortic stenosis at extreme risk for surgery. J Am Coll Cardiol 2014; 63:1972-81.

19. Kodali SK, Williams MR, Smith CR, et al; PARTNER Trial Investigators. Two-year outcomes after transcatheter or surgical aortic-valve replacement. N Engl J Med 2012; 366:1686-95.

20. Adams DH, Popma JJ, Reardon MJ. Transcatheter aortic-valve replacement with a self-expanding prosthesis. N Engl J Med 2014; 371:967-8.

21. Leon MB, Smith CR, Mack MJ, et al; PARTNER 2 Investigators. Transcatheter or surgical aortic-valve replacement in intermediate-risk patients. N Engl J Med 2016; 374:1609-20.

22. Dvir D, Webb JG, Bleiziffer S, et al; Valve-in-Valve International Data Registry Investigators. Transcatheter aortic valve implantation in failed bioprosthetic surgical valves. JAMA 2014; 312:162-70.

23. Chiam PT, Ewe SH, Soon JL, et al. Percutaneous transcatheter aortic valve implantation for degenerated surgical bioprostheses: the first case series in Asia with one-year follow-up. Singapore Med J 2016; 57:401-5.

24. See J, Amora JL, Lee S, et al. Non-fluoroscopic navigation systems for radiofrequency catheter ablation for supraventricular tachycardia reduce ionising radiation exposure. Singapore Med J 2016; 57:390-5.

25. Andreassi MG, Piccaluga E, Guagliumi G, et al. occupational health risks in cardiac catheterization laboratory workers. Circ Cardiovasc Interv 2016; 9(4). pii:e003273.

26. Leong KT, Goh PP, Chang BC, Lingamanaicker J. Heart failure cohort in Singapore with defined criteria: clinical characteristics and prognosis in a multi-ethnic hospital-based cohort in Singapore. Singapore Med J 2007; 48:408-14.

27. Desai AS, Stevenson LW. Rehospitalization for heart failure: predict or prevent? Circulation 2012; 126:501-6.

28. Costanzo MR, Guglin ME, Saltzberg MT, et al; UNLOAD Trial Investigators. Ultrafiltration versus intravenous diuretics for patients hospitalized for acute decompensated heart failure. J Am Coll Cardiol 2007; 49:675-83.

29. Teo LY, Lim CP, Neo CL, et al. Ultrafiltration in patients with decompensated heart failure and diuretic resistance: an Asian centre's experience. Singapore Med J 2016; 57:378-83.

30. Chia PL, Foo D. Overview of implantable cardioverter defibrillator and cardiac resynchronisation therapy in heart failure management. Singapore Med J 2016; 57:354-9. 